\title{
Synthesis of Substituted Thioureas and Their Sulfur Heterocyclic Systems of $p$-Amino Salicylic Acid as Antimycobacterial Agents
}

\author{
Mohammed Saleh I. T. Makki, Reda M. Abdel-Rahman, \\ Hassan M. Faidallah, and Khalid Ali Khan \\ Department of Chemistry, Faculty of Science, King Abdulaziz University, P.O. Box 80203, Jeddah 21589, Saudi Arabia \\ Correspondence should be addressed to Hassan M. Faidallah; hfaidallahm@hotmail.com
}

Received 14 April 2012; Revised 21 July 2012; Accepted 23 July 2012

Academic Editor: Theocharis C. Stamatatos

Copyright (C) 2013 Mohammed Saleh I. T. Makki et al. This is an open access article distributed under the Creative Commons Attribution License, which permits unrestricted use, distribution, and reproduction in any medium, provided the original work is properly cited.

\begin{abstract}
A series of new $\mathrm{N}, \mathrm{N}^{\prime}$-substituted thioureas $(\mathbf{2}, \mathbf{6}$, and $\mathbf{8})$ and their sulfur heterocycles as thiobarbituric acids $(\mathbf{3}, \mathbf{4}$, and $\mathbf{7})$, 2thioxothiazoliodin-4-one (10), thiazolidin-4-one (11), 1,2,4-triazol-5-thione (14), and 1,3,4-thiadiazole (15) of $p$-Amino salicylic acid (PAS) have been synthesized from treatment with dithiocarbazinate (1, 5 and 12) followed by heterocyclization with dimethyl malonate, chloroacetic acid, and/or trifluoroacetic anhydride. The structures of the newly synthesized compounds were substantiated with IR, ${ }^{1} \mathrm{H}$, and ${ }^{13} \mathrm{C}$ NMR spectral data and elementary microanalyses. The in vitro antitubercular activity of synthesized compounds against $M$. tuberculosis strain H37Rv showed moderate-to-good activity.
\end{abstract}

\section{Introduction}

Infection of Mycobacterium tuberculosis is more prevalent in the world today than at any other time in human history [1]. Globally, more than one-third of the world's population is infected with the bacteria that cause TB, and each year approximately 9 million people become ill with the disease, and 2 million of those die [2-5]. TB is the second leading cause of death from an infectious disease worldwide. The frequent coinfection of TB in HIV patients further complicates the selection of an appropriate treatment regimen. In recent years, multidrug resistant (MDR-TB) and extensively drug-resistant (XDR-TB) tuberculosis strains emerged and tuberculosis is considered as one of the most challenging threats to global health $[6,7]$.

During the last few years, chemical research developed a simple, safe, and efficient method to synthesize new classes of compounds active against $M$. tuberculosis [8-10]. Aminosalicylic acid (PAS) was introduced as an antitubercular medicine in 1948. It was the second antibiotic found to be effective in the treatment of tuberculosis, after streptomycin. PAS formed part of the standard treatment for tuberculosis prior to the introduction of rifampicin and pyrazinamide.
Its potency is less than that of the current five firstline drugs (isoniazid, rifampicin, ethambutol, pyrazinamide, and streptomycin) for treating tuberculosis, but it is still useful in the treatment of multidrug-resistant tuberculosis. PAS is always used in combination with other anti-TB drugs.

In a recent study, a salicylic acid analog (Figure 1) has been found to show mycobacterium protein tyrosine phosphatase B inhibiting activity. This is relatively a new concept in which the growth of mycobacteria is arrested by a pharmacological activation of the xenophagic pathway [11]. This analog of salicylic acid has provided an innovative therapeutic starting point for the treatment of TB, including MDR and XDR forms, that is not only complementary, but also synergistic with current drugs. It is anticipated that such combination therapy will result in the shorter duration of treatment and recovery time for the TB patient. In an extension of our previous study, in the area of synthesis of drugs, semidrugs and bioactive compounds for the treatment of infectious diseases [12-18], the present work aims at the synthesis of some new thiourea and sulphur-containing heterocyclic systems of $p$-amino salicylic acid as possible antitubercular agents. 
<smiles>O=C(Cn1cc(-c2c(-c3ccccc3)oc3cc(O)c(C(=O)O)cc23)nn1)Nc1ccc(N2CCOCC2)cc1</smiles>

Benzofuran salicylic acid (1-A09)

FIGURE 1

\section{Experimental}

Melting points were determined in an electrothermal Bibby Stuart scientific melting point SMP (US). The IR spectra recorded for $\mathrm{KBr}$ discs on a Perkin Elmer spectrum RXI FT-IR systems no. 53529. ${ }^{1} \mathrm{H} /{ }^{13} \mathrm{C}-\mathrm{NMR}$ was determined for solution in deuterated DMSO with a Bruker DPX-400-FT using TMS as an internal standard solvent (chemical shifts in $\delta$, ppm). Mass spectra were measured on a GCMS-Q 1000-Ex spectrometer. Microanalyses (C, H, N, S, F, and $\mathrm{Cl}$ ) elements were performed by the Microanalyses Centre of Cairo University, Egypt. The antituberculosis activity was carried out in National Institute of Allergy and Infection Diseases' Southern Research Institute, Gillis W. Long Hansen's Disease Center, Colorado State University, Birmingham, AL' USA.

General Procedure for the Synthesis of Potassium Dithiocarbamates $(\mathbf{1}, 5$, and 12). Carbon disulfide $(0.01 \mathrm{~mol})$ was added slowly with vigorous stirring to a mixture of the appropriate sulfa drug and ethanolic potassium hydroxide $(20 \mathrm{~mL}, 5 \%)$ at room temperature. The stirring was continued for $3 \mathrm{~h}$ and the precipitated potassium salt was filtered, washed with ether, dried and used for the next step without further purification.

General Procedure for the Synthesis of 2-Hydroxyl-4(Substituted-Thioureido) Benzoic Acid (2a, b \& 6) and 2-Hydroxy4-[(4-Pyridoyl) Thiosemicarbazido]]benzoic Acid (13). A mixture of PAS $(0.01 \mathrm{~mol})$ and the appropriate potassium dithiocarbamate $(0.01 \mathrm{~mol})$ in ethanol $(100 \mathrm{~mL})$ was refluxed for $2 \mathrm{~h}$. The solid which separated on cooling was filtered, washed with water, dried, and recrystallized from ethanol.

2a. Yield 78\%; yellow powder, m.p. $230-231^{\circ} \mathrm{C}$ (decomp); IR $\left(v \mathrm{~cm}^{-1}\right) 3410,3380(2 \mathrm{OH}), 3320,3210(\mathrm{NH}), 3030$ $(\mathrm{Ar}-\mathrm{H}), 1666(\mathrm{C}=\mathrm{O}), 1589(\mathrm{C}=\mathrm{N}), 1350\left(\mathrm{SO}_{2} \mathrm{NH}\right), 1266$ (H-bonding), $1201(\mathrm{C}=\mathrm{S}), 810,800(\mathrm{Ar}-\mathrm{CH}),{ }^{1} \mathrm{HNMR}(\delta$ ppm): $11.38(\mathrm{~s}, 1 \mathrm{H}, \mathrm{OH}), 8.80\left(\mathrm{~s}, 1 \mathrm{H}, \mathrm{NHSO}_{2}\right), 8.51(\mathrm{~s}$, $1 \mathrm{H}, \mathrm{NHCS}), 7.81-8.36(\mathrm{~m}, 3 \mathrm{H}$ pyrimidine-H), 7.2-7.01 (m, $7 \mathrm{H}$, aromatic H) ${ }^{13} \mathrm{CNMR}(\delta$-ppm): 206.5 (CS) $172.4(\mathrm{CO})$, 110.6, 157.4, 169.3 (pyrimidine-C) 112.5, 113.2, 117.6, 125.6, 125.8, 130.2, 135.4, 142.6, 153.3, 158.7 (Ar-C); Anal. Calcd.
TABLE 1: Antimycobacterial activity versus M. fortuitum ATCC 6841 and primary antituberculosis activity screening results.

\begin{tabular}{lccc}
\hline Compd. no. & $\mathrm{MIC} / \mu \mathrm{g} \mathrm{mL} \mathrm{a}^{\mathrm{a}}$ & $\mathrm{MIC} \mu \mathrm{g} \mathrm{mL}^{\mathrm{b}}$ & Inhibition $^{\mathrm{c}}(\%)$ \\
\hline $\mathbf{2 b}$ & 64 & $>12.5$ & 85 \\
$\mathbf{3 b}$ & 64 & $>12.5$ & 87 \\
$\mathbf{4}$ & $>128$ & $<12.5$ & 95 \\
$\mathbf{6}$ & 64 & $>12.5$ & 85 \\
$\mathbf{7}$ & 64 & $>12.5$ & 88 \\
$\mathbf{8 a}$ & 64 & $<12.5$ & 90 \\
$\mathbf{9 b}$ & $>128$ & $<12.5$ & 92 \\
$\mathbf{1 3}$ & $>128$ & $<12.5$ & 96 \\
$\mathbf{1 4}$ & $>128$ & $>12.5$ & 90 \\
$\mathbf{1 5}$ & $>128$ & $>12.5$ & 90 \\
\hline
\end{tabular}

${ }^{\mathrm{a}}$ MIC affecting $M$. fortuitum ATCC6841.

${ }^{\mathrm{b}} \mathrm{MIC}$ affecting $M$. tuberculosis $\mathrm{H}_{37} \mathrm{Rv}$.

${ }^{c}$ Reduction of mycobacterial growth using $M$. tuberculosis $\mathrm{H}_{37} \mathrm{Rv}$ using $12.5 \mu \mathrm{g} / \mathrm{mL}$ concentration of compounds.

$\mathrm{C}_{18} \mathrm{H}_{15} \mathrm{~N}_{5} \mathrm{~S}_{2} \mathrm{O}_{5}$ (445): C, 48.53; H, 3.37; N, 15.73; S, 14.38 . Found: C, 48.38; H, 3.33; N, 15.54; S, 14.09.

2b. Yield 79\%, pale yellow powder, m.p. $180-182^{\circ} \mathrm{C}$ (decomp.); IR $\left(v \mathrm{~cm}^{-1}\right)$ 3424, $3333(2 \mathrm{OH}), 3074,3035$ $(\mathrm{Ar}-\mathrm{H}), 1670(\mathrm{C}=\mathrm{O}), 1588,1572(2 \mathrm{C}=\mathrm{N}), 1334\left(\mathrm{NHSO}_{2}\right)$, $1189(\mathrm{C}=\mathrm{S})$ 818, 795 (Ar-CH). MS (\%): $415\left(\mathrm{M}^{+}-\mathrm{CO}_{2}\right.$, 48.13), 371 (18.8), 215 (11.11), 156 (5.51), 151 (100), 92 (48.51). Anal. Calcd. $\mathrm{C}_{19} \mathrm{H}_{17} \mathrm{~N}_{5} \mathrm{~S}_{2} \mathrm{O}_{5}$ (459): C, 49.67; H, 3.70; N, 15.25; S, 13.94. Found: C, 49.29; H, 3.66; N, 15.08; S, 13.75 .

6. Yield $88 \%$; yellow crystalline, m.p. 196-197 $\mathrm{C}$ (decomp); IR $\left(v \mathrm{~cm}^{-1}\right)$ 3400-3310 (2 OH), $3210(\mathrm{NH}), 3042,3035(\mathrm{Ar}-\mathrm{H})$, 2936, 2888 (aliphatic CH), 1760, $1677(2 \mathrm{C}=\mathrm{O}), 1609(\mathrm{C}-\mathrm{N})$, 1492, 1412 (deformation $\mathrm{CH}_{3}$ ), 1345, 1310 (NCSN), $1281(\mathrm{H}-$ bonding), $1186(\mathrm{C}=\mathrm{S}), 882,794(\mathrm{Ar}-\mathrm{CH}) .{ }^{1} \mathrm{HNMR}(\delta$-ppm): 2.55 (s, 3H, $\left.\mathrm{CH}_{3}\right), 4.32\left(\mathrm{~s}, 3 \mathrm{H}, \mathrm{CH}_{3}-\mathrm{N}\right), 7.65-7.38(\mathrm{~m}, 8 \mathrm{H}$, Ar-H), 8.31 (s, 1H, NH), 11.81 (s, 1H, OH). ${ }^{13} \mathrm{CNMR}(\delta-$ ppm): $15.5\left(\mathrm{CH}_{3}\right), 35.3\left(\mathrm{~N}-\mathrm{CH}_{3}\right) 199.9$ (CS), 171.7 (CO), 162.84 (CO), 108.7, 110.2, 113.3, 114.1, 118.9, 129.5, 130.3, 131.4, 131.3, 142.3, 146.7, 158.5. Anal. Calcd. $\mathrm{C}_{19} \mathrm{H}_{18} \mathrm{~N}_{4} \mathrm{SO}_{4}$ (398): C, 57.28; H, 4.52; N, 14.07; S, 8.04. Found: C, 56.78; H, $4.46 ; \mathrm{N}, 13.91 ; \mathrm{S}, 7.95$.

13. Yield $89 \%$, orange powder, m.p. $133-134^{\circ} \mathrm{C}$ (decomp.); IR $\left(v \mathrm{~cm}^{-1}\right) 3494(\mathrm{OH}), 3386(\mathrm{NH}), 2535(\mathrm{SH}), 1641(\mathrm{C}=\mathrm{O})$, $1615(\mathrm{C}=\mathrm{N}), 1352$, (NCS), 1293 (H-bonding), $1227(\mathrm{~N}-\mathrm{N})$, $1194(\mathrm{C}-\mathrm{S}), 881,809,768(\mathrm{Ar}-\mathrm{CH}) .{ }^{1} \mathrm{HNMR}(\delta$-ppm) $: 5.85$ (s, 1H, OH), 6.4-7.2 (m, 3H of aryl), 7.7, 7.9 (each dd, $2 \mathrm{H}, 2 \mathrm{H}$ of pyridine), 9.2, 8.8, $8.2(3 \mathrm{~s}, 3 \mathrm{H}, 3 \mathrm{NH}), 11.54(\mathrm{~s}$, $1 \mathrm{H}, \mathrm{OH}) .{ }^{13} \mathrm{CNMR}(\delta$-ppm): $187.6(\mathrm{CS}), 172.1(\mathrm{CO}), 163.5$ (CO), 112.3, 114.2, 117.8, 122.4, 131.4, 142.5, 144.8, 150.3, 159.1 (Ar-C); $\mathrm{MS}(\%): 288\left(\mathrm{M}^{+}-\mathrm{CO}_{2}, 10.11\right), 151$ (100), 107 (15.10), 106 (38.10), 78 (12.98). Anal. Calcd. $\mathrm{C}_{14} \mathrm{H}_{12} \mathrm{~N}_{4} \mathrm{SO}_{4}$ (332): C, 50.60; H, 3.61; N, 16.86, S, 9.63. Found: C, 49.95; H, $3.54 ; \mathrm{N}, 16.66 ; \mathrm{S}, 9.63$. 


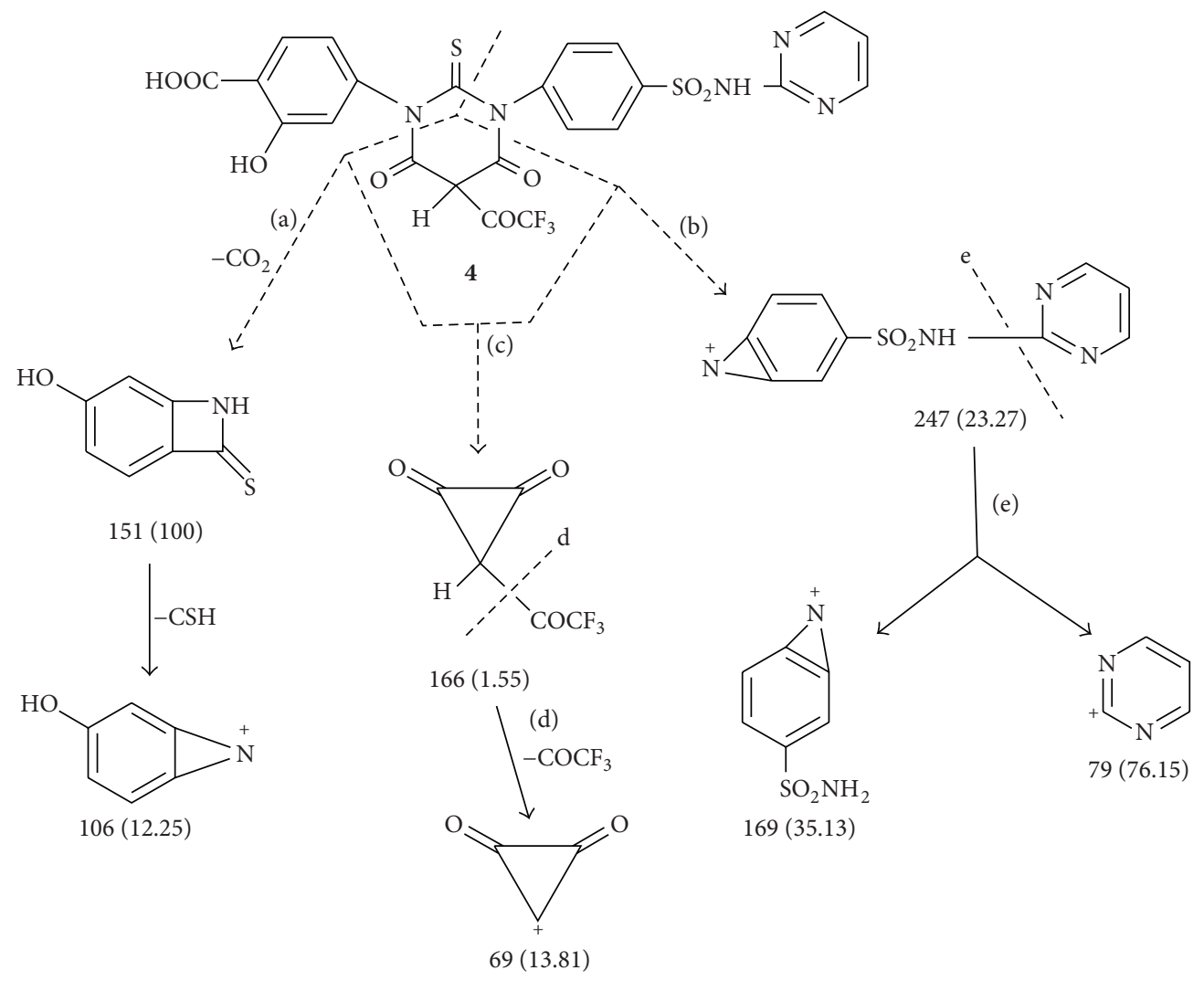

FIGURE 2: Mass fragmentation pattern of compound 4.

TABLE 2: Level 2 antituberculosis acidity assay results of $4,9 b, 13$, and 14 (Level 2 assay).

\begin{tabular}{lccc}
\hline Compd. no. & $\mathrm{MIC} / \mu \mathrm{g} \mathrm{mL}$ & $\mathrm{IC}_{50}(\mu \mathrm{g} \mathrm{mL})$ & $\mathrm{SI}$ \\
\hline $\mathbf{4}$ & 6.25 & $>10$ & 1.5 \\
$\mathbf{9 b}$ & $>12.5$ & - & - \\
$\mathbf{1 3}$ & $>12.5$ & - & - \\
$\mathbf{1 4}$ & 6.25 & 10 & 1.6 \\
\hline
\end{tabular}

The assay was performed using BACTEC in which MIC of RMP was found as $0.125 \mu \mathrm{g} / \mathrm{mL}$.

3-(Aryl/hetaryl)-1-(2-hydroxy benzoic acid)thiobarbituric acid (3a, 3b \& 7). A mixture of the appropriate thiourea derivative $(0.01 \mathrm{~mol})$ in THF $(100 \mathrm{~mL})$ was refluxed with dimethyl malonate $(0.01 \mathrm{~mol})$ for $12 \mathrm{~h}$. The obtained solid which separated on cooling was filtered, washed with ethanol, and recrystallized from THF.

3a. Yield 68\%; yellow powder, m.p. $240-241^{\circ} \mathrm{C}$ (decomp); IR $\left(\nu \mathrm{cm}^{-1}\right) 3420(\mathrm{OH}), 3352(\mathrm{OH}), 3258\left(\mathrm{NHSO}_{2}\right), 3074,3037$ $(\mathrm{Ar}-\mathrm{H}), 2936,2869$ (str. $\left.\mathrm{CH}_{2}\right) 1651(\mathrm{C}=\mathrm{O}), 1577(\mathrm{C}=\mathrm{N})$, 1439 (bending $\mathrm{CH}_{2}$ ), 1324 (NCSN), 1261 (H-bonding), 1149 $(\mathrm{C}=\mathrm{S}), 841,820(\mathrm{Ar}-\mathrm{CH}) .{ }^{1} \mathrm{HNMR}(\delta$-ppm): 3.17 (pyrimidine $\left.\mathrm{CH}_{2}\right), 6.5(\mathrm{~d}, 1 \mathrm{H}$, thiazole $\mathrm{H}-5), 7.5(\mathrm{~d}, 1 \mathrm{H}$, thiazole $\mathrm{H}-$ 5), 7.7-8.1(m, 7H, Ar-H), $11.3(\mathrm{~s}, 1 \mathrm{H}, \mathrm{OH}), 11.2(\mathrm{~s}, 1 \mathrm{H}$, $\mathrm{OH}) .{ }^{13} \mathrm{CNMR}\left(\delta\right.$-ppm): $34.5\left(\mathrm{CH}_{2}\right) 108.4,138.4,171.1$
(thiazole-C), 107.1, 112.5, 113.8, 120.4, 125.7, 131.9, 134.3, 144.8, 147.6, 159.8, 179.5 (CS), 164.9 (CO). Anal. Calcd. $\mathrm{C}_{21} \mathrm{H}_{15} \mathrm{~N}_{5} \mathrm{~S}_{2} \mathrm{O}_{7}$ (513): C, 49.12; H, 2.92; N, 13.64, S, 12.47. Found: C, 48.85; H, 2.93; N, 13.38; S, 12.21.

3b. Yield 66\%, faint yellow; m.p. 214-215 $\mathrm{C}$ (decomp.); IR $\left(v \mathrm{~cm}^{-1}\right) 3410(\mathrm{OH}), 3320(\mathrm{OH}), 3250\left(\mathrm{NHSO}_{2}\right), 3060$ $(\mathrm{Ar}-\mathrm{H}), 2916,2899$ (str. $\left.\mathrm{CH}_{2}\right) 1661(\mathrm{C}=\mathrm{O}), 1587(\mathrm{C}=\mathrm{N})$, 1488, 1441 (bending $\mathrm{CH}_{2}$ ), 1351 (NCSN), 1271 (H-bonding), $1185(\mathrm{C}=\mathrm{S}), 821,808$ (Ar-CH). MS(\%): $415\left(\mathrm{M}^{+}-\mathrm{CO}_{2}\right.$, $-\mathrm{C}_{3} \mathrm{H}_{2} \mathrm{O}_{2}$ ) (12.25), 156 (55.13), 151 (100), 108 (13.1). Anal. Calcd. $\mathrm{C}_{22} \mathrm{H}_{17} \mathrm{~N}_{5} \mathrm{~S}_{2} \mathrm{O}_{7}$ (527): C, 50.09; H, 3.22; N, 13.28, S, 12.14. Found: C, 49.69; H, 3.8; N, 13.13; S, 12.06 .

7. Yield 65\%; yellow powder; m.p. $131-132^{\circ} \mathrm{C}$ (decomp.); IR $\left(v \mathrm{~cm}^{-1}\right) 3305(\mathrm{OH}), 3489(\mathrm{OH}), 3250\left(\mathrm{NHSO}_{2}\right), 2977,2899$ (str. $\left.\mathrm{CH}_{2}, \mathrm{CH}_{3}\right), 1650(\mathrm{C}=\mathrm{O}), 1616,1608(\mathrm{C}=\mathrm{C}, \mathrm{C}=\mathrm{N}), 1454$ (deform $\mathrm{CH}_{2}$ ), 1441 (bending $\mathrm{CH}_{2}$ ), 1368 (NCSN), 1279 (H-bonding), 1219 (C-N), $1194(\mathrm{C}=\mathrm{S}), 877,867$ (Ar-CH). MS(\%): $468\left(\mathrm{M}^{+}+21.01\right), 279$ (5.90), 187 (23.11), 151 (100), 84 (1.18). ${ }^{1} \mathrm{HNMR}(\delta$-ppm): 2.2 (s, 3H, Me-C), 3.5 (s, 3H, Me-N), 5.8 (s, 1H, OH), 6.1 (s, 1H, Pyrimidine- $\mathrm{H}$ ), 6.95-7.9 (m, 8H, aromatic $\mathrm{H}), 10.6,(\mathrm{~s}, 1 \mathrm{H}, \mathrm{OH}), 14.2$ (s, $1 \mathrm{H}$, pyrimidine $\mathrm{OH}) .{ }^{13} \mathrm{CNMR}(\delta$-ppm): $15.3(\mathrm{Me}-\mathrm{C}), 35.1$ $(\mathrm{Me}-\mathrm{N}), 68.33$ (pyrimidine $\mathrm{CH}=$ ), 105.4 (pyrazolidinone $\mathrm{H}$ 4), 129.4 (pyrazolidinone $\mathrm{H}-3$ ), 107.3, 112.2, 112.9, 113.3, 118.6, 129.1, 131.8, 142.2, 144.5, 159.0 (Ar-C), 160.76 (CO), 


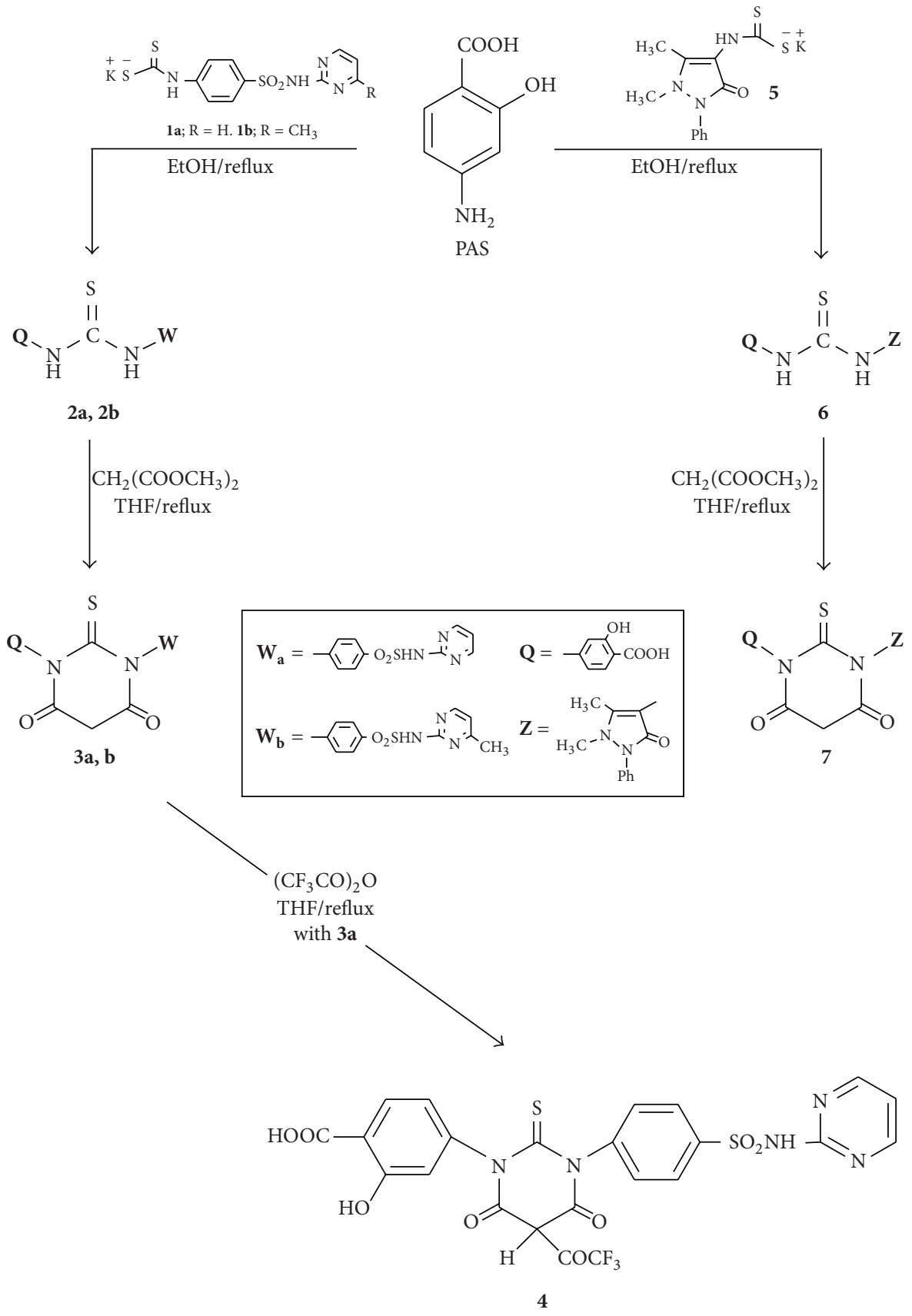

SCHEME 1

164.1 (CO), 174.2 (CO). Anal. Calcd. $\mathrm{C}_{22} \mathrm{H}_{18} \mathrm{~N}_{4} \mathrm{SO}_{6}$ (466): C, 56.65; H, 3.86; N, 12.01; S, 6.86. Found: C, 56.35; H, 3.79; N, $11.76 ; \mathrm{S}, 6.79$.

4-\{4,6-Dioxo-3-[4-(pyrimidin-2-ylsulfamoyl)phenyl]-2-thioxocyclohexyl\}-2-hydroxybenzoic acid (4). Compound 3 (1 gm) was refluxed with a mixture of trifluoroacetic anhydride $(5 \mathrm{~mL})$ and trifluoroacetic acid $(5 \mathrm{~mL})$ for $1 \mathrm{~h}$. The precipitated solid, which separated on cooling, was filtered and recrystallized from dioxan as faint yellow needles. Yield 55\%; m.p. $211-212^{\circ} \mathrm{C}$ (decomp); $\operatorname{IR}\left(v \mathrm{~cm}^{-1}\right): 3310 \mathrm{w},(\mathrm{OH})$,
3103 (w, NH), 3035 (Ar-CH), 2877 (aliphatic CH) 1714, $1671(2 \mathrm{C}=\mathrm{O}), 1604(\mathrm{C}=\mathrm{N}), 1525(\mathrm{C}=\mathrm{N}), 1499$ (deform. $\mathrm{CH})$, 1345 (NCSN), $1319\left(\mathrm{NHSO}_{2}\right), 1280(\mathrm{C}-\mathrm{N}), 1181(\mathrm{C}=\mathrm{S})$, 836, 826, 816 (Ar-CH \& hetero-CH). MS(\%): $610\left(\mathrm{M}^{+}+\right.$ 1, 1.11$) 247$ (23.27), 169 (35.15), 166 (1.55), 151 (100), 106 (12.25), 79 (76.15), 69 (13.81). ${ }^{1} \mathrm{HNMR}(\delta$-ppm): 4.82 (s, $1 \mathrm{H}, \mathrm{CH}-\mathrm{CO}), 6.95(\mathrm{~m}, 1 \mathrm{H}$, pyrimidine $\mathrm{H}-5), 8.43$ (dd, $2 \mathrm{H}$, pyrimidine H-4 \& H-6), 7.32-7.94 (m, 7H, ArH), 8.23 (s, 1H, of pyrimidine), $11.2(\mathrm{~s}, 1 \mathrm{H}, \mathrm{OH}) .{ }^{13} \mathrm{CNMR}(\delta$-ppm): 43.4 (CHCO) 110.3, 157.3, 166.4 (pyrimidine-C), 107.4, 112.3, 113.8, 120.4, 125.6, 131.7, 134.9, 144.0, 148.5, 159.5 (Ar-C), 


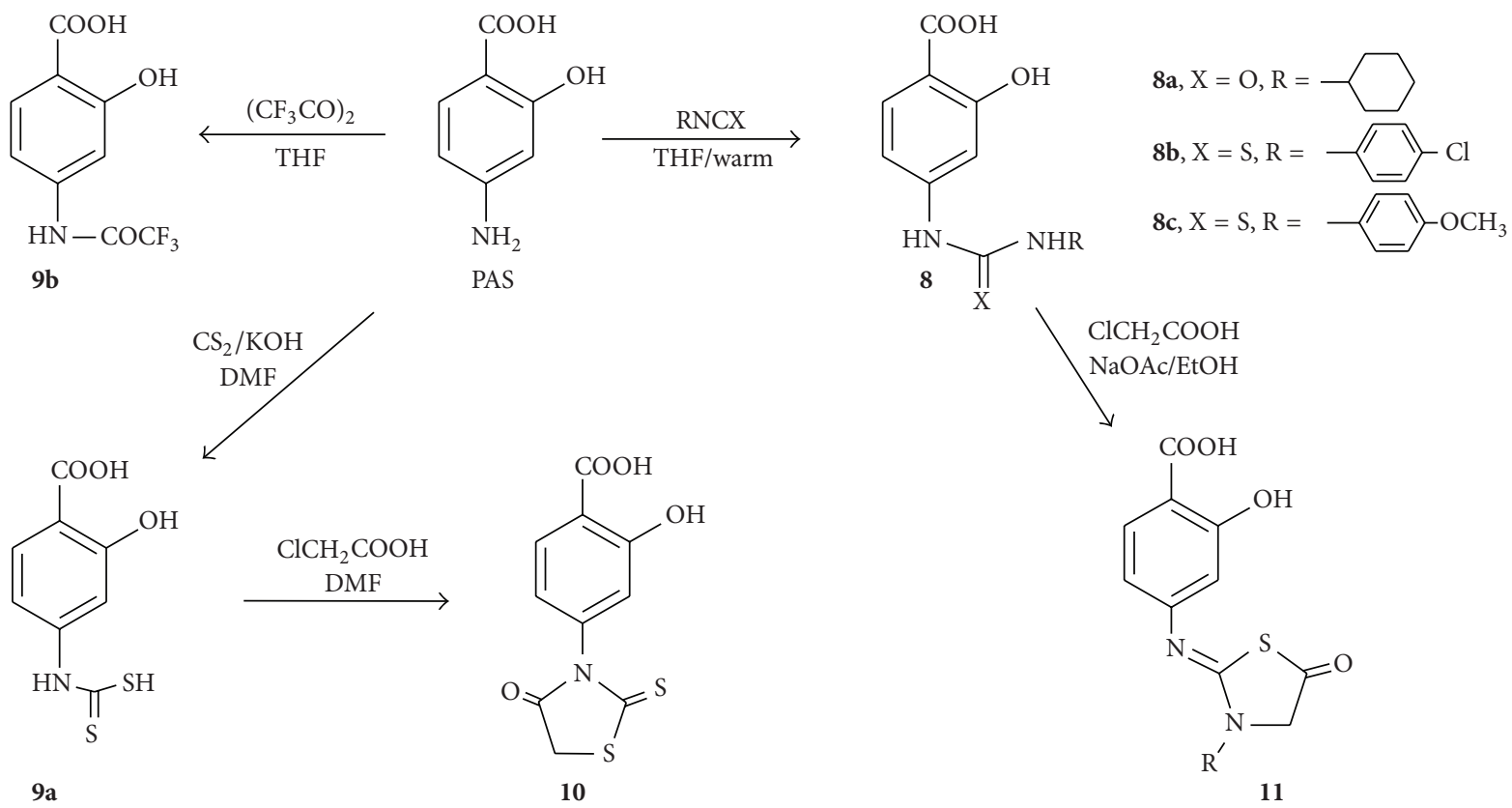

SCHEME 2

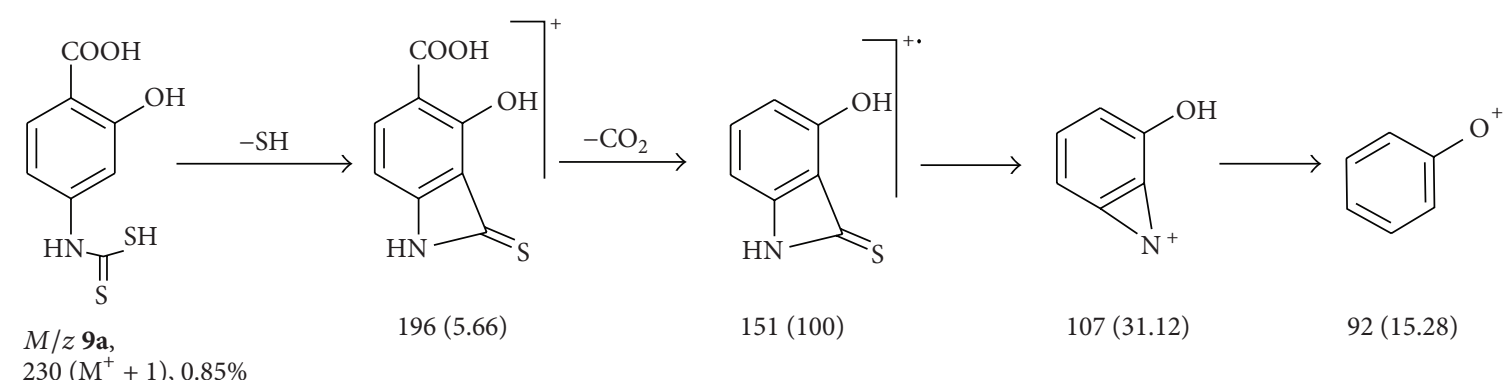

FigURE 3: Mass fragmentation pattern of 9a.

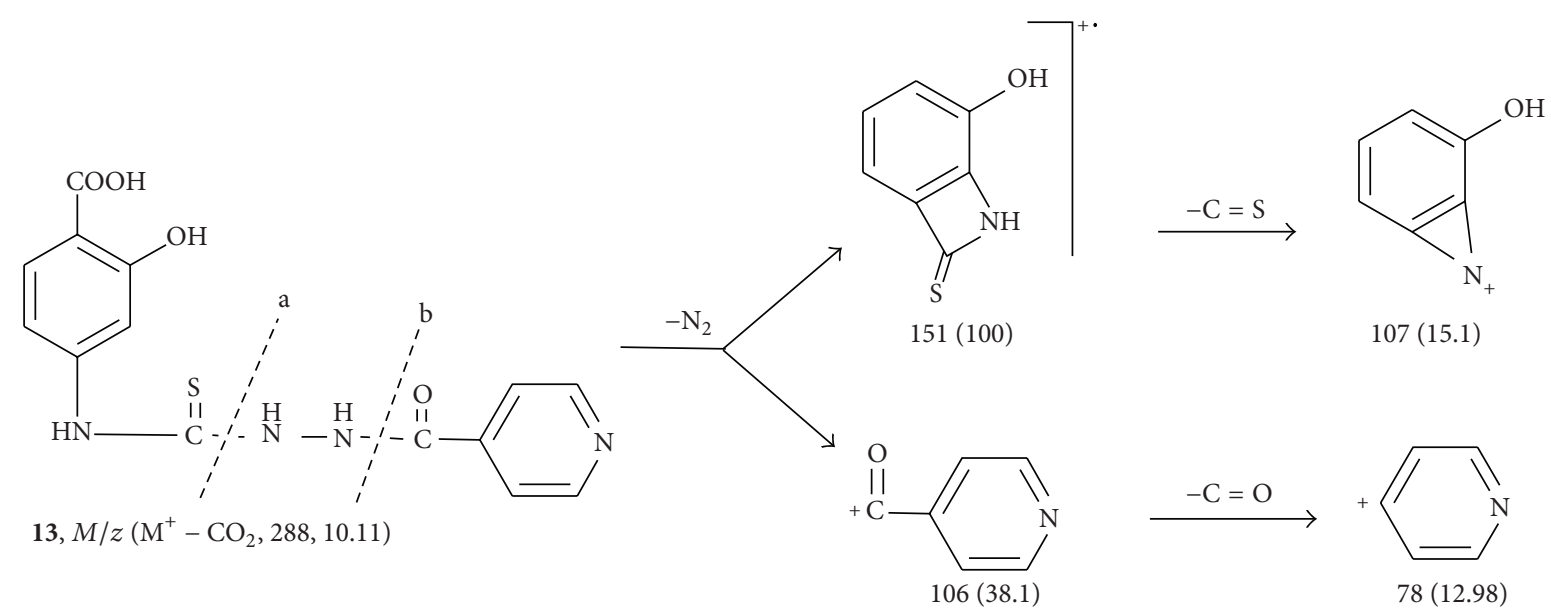

FIGURE 4: Mass fragmentation pattern of compound 13. 


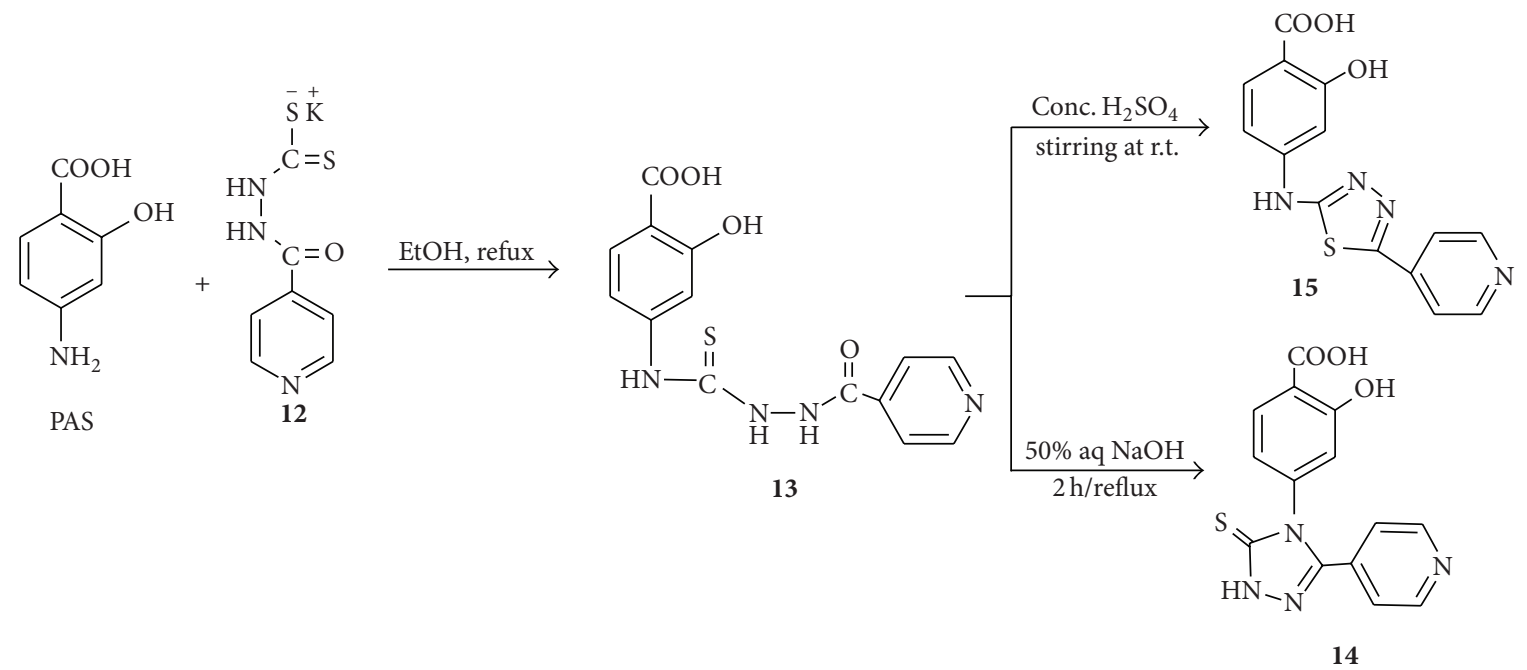

SCHEME 3

$127.9\left(\mathrm{CF}_{3}\right), 168.9,172.5$ (2CO), $204.1\left(\mathrm{CO}-\mathrm{CF}_{3}\right), 184(\mathrm{CS})$. Anal. Calcd. $\mathrm{C}_{23} \mathrm{H}_{14} \mathrm{~N}_{5} \mathrm{~S}_{2} \mathrm{~F}_{3} \mathrm{O}_{8}$ (609): C, 45.32; H, 2.29; N, 11.49, S, 10.50, F; 9.35. Found: C, 44.89; H, 2.26; N, 11.22; S, 10.38, F; 9.25.

2-Hydroxyl-4-(Substituted-Ureido- and Thioureido)benzoic Acid $(\mathbf{8 a}-\mathbf{c})$. A mixture of PAS $(0.01 \mathrm{~mol})$ and the appropriate isocyanate or isothiocyanates derivative $(0.01 \mathrm{~mol})$ in THF $(100 \mathrm{~mL})$ was warmed at $100^{\circ} \mathrm{C}$ for $2 \mathrm{~h}$, then cooled to room temperature. The precipitate solid obtained was filtered and recrystallized from ethanol.

8a. Yield $90 \%$, faint yellow crystals; m.p. 203-204 ${ }^{\circ} \mathrm{C}$ (decomp); IR $\left(v \mathrm{~cm}^{-1}\right) 3446(\mathrm{OH}), 3301(\mathrm{NH}), 3010(\mathrm{Ar}-\mathrm{CH}), 2900$, 2840 (str. CH) $1675(\mathrm{C}=\mathrm{O}), 1655(\mathrm{C}=\mathrm{O}), 1525(\mathrm{C}=\mathrm{N}), 1394$ (NCSN), 1286 (H-bonding), $1230(\mathrm{C}-\mathrm{N}), 831,804(\mathrm{Ar}-\mathrm{CH})$. Anal. Calcd. $\mathrm{C}_{14} \mathrm{H}_{18} \mathrm{~N}_{2} \mathrm{O}_{4}$ (278): C, 60.43; H, 6.47; N, 10.07. Found: C, 59.79; H, 6.38; N, 9.95.

8b. Yield 88\%; orange crystalline, m.p. $164-165^{\circ} \mathrm{C}$ (decomp.); IR $\left(v \mathrm{~cm}^{-1}\right) 3399(\mathrm{OH}), 3211(\mathrm{NH}), 3009(\mathrm{Ar}-\mathrm{CH}), 1660$ $(\mathrm{C}=\mathrm{O}), 1655(\mathrm{C}=\mathrm{O}), 1355$ (NCSN), 1265 (H-bonding), 1225 $(\mathrm{C}-\mathrm{N}), 1195 \mathrm{C}=\mathrm{S}), 830,810(\mathrm{Ar}-\mathrm{CH}), 710(\mathrm{C}-\mathrm{Cl})$. Anal. Calcd. $\mathrm{C}_{14} \mathrm{H}_{11} \mathrm{~N}_{2} \mathrm{SClO}_{3}$ (323): C, 52.01; H, 3.40; N, 8.66, S, 9.90, Cl; 11.12. Found: C, 51.88; H, 3.35; N, 8.54; S, 9.83, Cl; 11.00 .

8c. Yield 91\%; yellowish crystalline; m.p. $161-162^{\circ} \mathrm{C}$ (decomp.); IR $\left(v \mathrm{~cm}^{-1}\right) 3674$ (b, OH), $3209(\mathrm{NH}), 3004$ (Ar-CH), 2900, 2837 (str. CH), $1655(\mathrm{C}=\mathrm{O}), 1620(\mathrm{C}=\mathrm{O})$, 1355 (CSNH), 1584, 1564 (C=C), 1459, 1416 (deform. Me), 1328 (NCSN), 1286 (H-bonding), $1230(\mathrm{C}-\mathrm{N}), 1165 \mathrm{C}=\mathrm{S})$, 1032 (-OMe), 865, 831, $804(\mathrm{Ar}-\mathrm{CH}) .{ }^{1} \mathrm{HNMR}(\delta$-ppm): 3.38 (s, 3H, -OMe), 6.39-7.81(m, 7H, ArH), 8.30 (s, 1H, OH phenolic), 9.96, 9.91 (each s, NH, NH), 11.5 (s, 1H, OH). ${ }^{13} \mathrm{CNMR}(\delta$-ppm): 112.2, 113.7, 114.4, 117.9, 126.4, 131.5, 133.3, 145.6, 158.2, 159.4, 179.15 (AR-C) 171.5(CO), 179.8
(CS). Anal. Calcd. $\mathrm{C}_{15} \mathrm{H}_{14} \mathrm{~N}_{2} \mathrm{SO}_{4}$ (318): C, 56.60; H, 4.40; N, 8.80; S, 10.06. Found: C, 55.98; H, 4.35; N, .8.60; S, 9.94.

4-Dithiocarboxyamino-2-Hydroxy Benzoic Acid (9a). Equimolar mixture of PAS and $\mathrm{CS}_{2}$ in ethanolic $\mathrm{KOH}(50 \mathrm{~mL}$, $5 \%)$ and DMF $(50 \mathrm{~mL})$ was warmed for 30 minutes and then cooled. The reaction mixture was acidified using dilute $\mathrm{AcOH}$. The resulting solid was filtered and recrystallized from methanol as orange needles. Yield 92\%; m.p. 141-142 ${ }^{\circ} \mathrm{C}$ (decomp). IR $\left(v \mathrm{~cm}^{-1}\right) 3492(\mathrm{OH}), 3385(\mathrm{NH}), 2970,2522$ $(\mathrm{SH}), 1800(\mathrm{C}=\mathrm{O}), 1615(\mathrm{C}=\mathrm{N}), 1284(\mathrm{NCS}), 1225(\mathrm{C}-\mathrm{N})$, 1197, $1106(\mathrm{C}-\mathrm{S}), 878,817$ (Ar-CH). MS (\%): $230\left(\mathrm{M}^{+}+1\right.$, 0.85) 196 (5.66), 151 (100), 107 (31.12), 92 (15.28). ${ }^{1} \mathrm{HNMR}$ $(\delta$-ppm): 5.98 (s, 1H, SH), 6.25-7.44 (m, 3H,ArH), 8.121 (s, $1 \mathrm{H}, \mathrm{NH}), 11.5$ (s, 1H, OH). ${ }^{13} \mathrm{CNMR}(\delta$-ppm): 106.5, 113.6, $117.8,131.7,144.5,158.4$ (Ar-C), 174.3 (CO), 198.2 (CS). Anal. Calcd. $\mathrm{C}_{8} \mathrm{H}_{7} \mathrm{NS}_{2} \mathrm{O}_{3}$ (229): C, 41.92; H, 3.05; N, 6.11; S, 27.94. Found: C, 41.72;H, 3.01; N, 6.06; S, 27.67.

4-Trifluoroacetylamino-2-hydroxybenzoic acid (9b). A mixture of PAS $(0.01 \mathrm{~mol})$ and trifluoroacetic anhydride $(0.01 \mathrm{~mol})$ in THF $(100 \mathrm{~mL})$ was warmed for $30 \mathrm{~min}$. and cooled. The produced solid was recrystallized from dioxan as faint yellow needles. Yield 88\%; m.p. 119-120 $\mathrm{C}$ (decomp); IR $\left(v \mathrm{~cm}^{-1}\right) 3410(\mathrm{OH}), 3150(\mathrm{NH}), 2710(\mathrm{OH}), 1701,1660$ (2C=O), 1280 (H-bonding) $1260(\mathrm{C}-\mathrm{F}), 810,805(\mathrm{Ar}-\mathrm{CH})$, 695 (C-F). MS (\%): $250\left(\mathrm{M}^{+}+1,5.11\right) 135$ (100), 107 (18.05), 93 (38.27). Anal. Calcd. $\mathrm{C}_{9} \mathrm{H}_{6} \mathrm{NF}_{3} \mathrm{O}_{4}$ (249): C, 43.37; $\mathrm{H}, 2.40$; N, 5.62; F, 22.89. Found: C, 42.89; H, 2.37; N, 5.49; F, 22.63.

2-Hydroxy-4-(4-oxo-2-thioxothiazolidin-3-yl)benzoic acid (10). A mixture of $9 \mathrm{a}(0.01 \mathrm{~mol})$ and chloroacetic acid $(0.01 \mathrm{~mol})$ in DMF $(100 \mathrm{~mL})$ was heated at $100^{\circ} \mathrm{C}$ for $4 \mathrm{~h}$. After being cooled to room temperature, the reaction mixture was poured on ice cold water and the separated solid product was filtered, washed with water, dried and recrystallized from THF as yellow needles. Yield 80\%; m.p. $>280^{\circ} \mathrm{C} ; \operatorname{IR}\left(v \mathrm{~cm}^{-1}\right): 3392(\mathrm{OH}), 2975$ (str. $\left.\mathrm{CH}_{2}\right), 1724$ 
(C=O), 1607 (C=C), 1492 (deform. $\mathrm{CH}_{2}$ ), 1371 (NCS), 1227 (H-bonding), $1160(\mathrm{C}=\mathrm{S}), 1078(\mathrm{C}-\mathrm{S}-\mathrm{C})$. MS (\%): $270\left(\mathrm{M}^{+}\right.$ $+1,1.55), 151$ (100), 72 (18.28). Anal. Calcd. $\mathrm{C}_{10} \mathrm{H}_{7} \mathrm{NS}_{2} \mathrm{O}_{4}$ (269): C, 44.60; H, 2.60; N, 5.20; S, 23.79. Found: C, 44.41; H, 2.57 ; N, 5.19; F, 23.55 .

\section{2-Hydroxy-4-(4-oxo-3-p-methoxyphenylthiazolidin-2-ylide-} neamino)benzoic acid (11). A mixture of $8 \mathrm{c}(0.01 \mathrm{~mol})$ and chloroacetic acid $(0.01 \mathrm{~mol})$ with anhydrous sodium acetate $(10 \mathrm{~g})$ and absolute ethanol $(50 \mathrm{~mL})$ was refluxed for $2 \mathrm{~h}$, cooled and then poured on ice. The solid obtained was filtered off and crystallized from ethanol to give 11. Yield 65\%, yellowish powder, m.p. 151-152 ${ }^{\circ} \mathrm{C}$ (decomp.); IR $\left(\nu \mathrm{cm}^{-1}\right): 3300(\mathrm{OH}), 2977-2837\left(\right.$ str. $\left.\mathrm{CH}_{2}\right), 1724(\mathrm{C}=\mathrm{O})$, $1597(\mathrm{C}=\mathrm{N}), 1454$ (deform. $\left.\mathrm{CH}_{2}\right), 1370$ (NCS), $1297(\mathrm{H}-$ bonding), $1145(\mathrm{C}-\mathrm{N}), 1140(\mathrm{C}=\mathrm{S}), 1075(\mathrm{C}-\mathrm{O}-\mathrm{Me}), 883$, $826(\mathrm{Ar}-\mathrm{CH}) .{ }^{1} \mathrm{HNMR}\left(\delta\right.$-ppm) $3.72\left(\mathrm{~s}, 3 \mathrm{H}, \mathrm{OCH}_{3}\right), 4.16(\mathrm{~s}$, $2 \mathrm{H}$, thiazolidine $\mathrm{H}-4), 6.55-7.89(\mathrm{~m}, 3 \mathrm{H}, \mathrm{ArH}) 10.89$ (s, $1 \mathrm{H}$, $\mathrm{OH}) .{ }^{13} \mathrm{CNMR}\left(\delta\right.$-ppm): $56.4\left(\mathrm{OCH}_{3}\right), 68.5$ (thiazolidinone C-4), 163.6 (thiazolidinone C-2) 109.2, 113.2, 114.5, 116.1, $132.2,135.4,150.2,153.2,160.4,172.07$ (CO), 194.7 (CO), $171.35,162.61,159.37,156.15,154.56,131.38,129.152$, $127.07,121.80,114.28,113.63,112.20,111.36,109.11$, 108.35, 104.49. Anal. Calcd. $\mathrm{C}_{17} \mathrm{H}_{14} \mathrm{~N}_{2} \mathrm{SO}_{5}$ (358): C, 56.98; H, 3.91; N, 7.82; S, 8.93. Found: C, 56.48; H, 3.86; N, .7.73; S, 8.48 .

2-Hydroxy-4-(3-(Pyridin-4-yl)-5-Thioxo-1H-1,2,4-Triazol4(5H)-yl)benzoic Acid (14). Compound 13 (1 gm) in aqueous $\mathrm{NaOH}(50 \mathrm{~mL} 5 \%)$ was warmed at $80^{\circ} \mathrm{C}$ for $30 \mathrm{~min}$, cooled then acidified by dilute hydrochloric acid. The isolated solid was filtered, washed with water, and recrystallized as faint yellow needles from THF. Yield 55\%; m.p. 199-200 ${ }^{\circ} \mathrm{C}$ (decomp); IR $\left(v \mathrm{~cm}^{-1}\right)$ : 3300-2942 (b, OH, NH), 2624 (SH), $1680(\mathrm{C}=\mathrm{O}), 1609,1572(\mathrm{C}=\mathrm{N}), 1346$ (NCS), 1282 (H-bonding), $1234(\mathrm{C}-\mathrm{N}), 1188(\mathrm{C}-\mathrm{S}), 883,(\mathrm{Ar}-\mathrm{CH})$. ${ }^{1} \mathrm{HNMR}(\delta$-ppm): 7.34-7.86 (m, 3H, Ar-H), 8.02, 8.76 (2d, each of $2 \mathrm{H}$, pyridine-H), $8.3(\mathrm{~s}, 1 \mathrm{H}, \mathrm{NH}), 11.6(\mathrm{~s}, 1 \mathrm{H}, \mathrm{OH})$. ${ }^{13}$ CNMR $(\delta$-ppm): 108.5, 111.9, 113.9, 121.5, 126.6, 130.7, 140.3, 143.2, 148.6, 155.9, 157.9, 166.7 (CS), 171.8 (CO). Anal. Calcd. $\mathrm{C}_{14} \mathrm{H}_{10} \mathrm{~N}_{4} \mathrm{SO}_{3}$ (314): C, 53.50; H, 3.18; N, 17.83; S, 10.19. Found: C, 52.28; H, 3.08; N, 17.63; S, 10.07.

2-Hydroxy-4-(5-pyridin-4-yl-[1,3,4] thiadiazol-2-ylamino) benzoic acid (15). To an ice-cooled stirred solution of conc., $\mathrm{H}_{2} \mathrm{SO}_{4}(1.0 \mathrm{~mL})$ was added in small portions of the thiosemicarbazide derivative $13(1 \mathrm{~g})$ over a period of $15 \mathrm{~min}$. Stirring was maintained for further $1 \mathrm{~h}$ at room temperature, then the reaction mixture was then poured onto ice. The resulting solid product was filtered, washed with water, dried, and recrystallized from dioxan as orange needles. Yield 62\%; m.p. 204-205 ${ }^{\circ} \mathrm{C}$ (decomp); IR $\left(\nu \mathrm{cm}^{-1}\right)$ : 3350-3150 (b, OH, NH), $1652(\mathrm{C}=\mathrm{O}), 1508(\mathrm{C}=\mathrm{N}), 1350$ (NCS), 1258 (H-bonding), 1214 (C-N), 1085 (C-S-C), 868, 850, 779 (hetero/aryl CH). ${ }^{1} \mathrm{HNMR}(\delta$-ppm): $7.51,8.25$ (m, $4 \mathrm{H}$, of pyridine), $8.14(\mathrm{~s}, 1 \mathrm{H}, \mathrm{NH}), 11.1(\mathrm{~s}, 1 \mathrm{H}, \mathrm{OH})$. ${ }^{13}$ CNMR $(\delta$-ppm): 102.4, 107.5, 122.4, 132.1, 136.3, 141.3,
$143.2,150.3,153.4,154.1,159.3,163.6,172.3$ (CO). Anal. Calcd. $\mathrm{C}_{14} \mathrm{H}_{10} \mathrm{~N}_{4} \mathrm{SO}_{3}$ (314): C, 53.50; H, 3.18; N, 17.83; S, 10.19. Found: C, 52.95; H, 3.13; N, .17.83; S, 10.08 .

\section{Results and Discussion}

3.1. Chemistry. A series of new 2-hydroxyl-4-(substituted thioureido) benzoic acid $(2,6 \& 8)$ were synthesized via the reaction of PAS with the appropriate potassium dithiocarbamates $(1,5)$ and addition of isocyanate/isothiocyanates to PAS in THF under reflux (Schemes 1 and 2). The IR spectra showed two absorption bands at $3209-3258 \mathrm{~cm}^{-1}$, and $3310-3674 \mathrm{~cm}^{-1}$ for the $\mathrm{HN}$ and $\mathrm{OH}$ groups, respectively, along with a characteristic thiourea carbonyl absorption at $1150-1195 \mathrm{~cm}^{-1}$. Their ${ }^{1} \mathrm{H}-\mathrm{NMR}$ spectra exhibited beside the aromatic protons a singlet of one proton intensity at $\delta$ 11.35-511.81. The structures of the above compounds were further confirmed from their ${ }^{13} \mathrm{C}$ NMR and MS data (Section 2).

Ring closure reactions of compounds 2 and $\mathbf{6}$ with dimethyl malonate in refluxing THF produced the corresponding 1,3-disubstituted thiobarbituric acids 3 and 7, respectively. Their IR spectra showed two $\mathrm{OH}$ absorption bands at $3305-3352 \mathrm{~cm}^{-1}$ and $3410-3489 \mathrm{~cm}^{-1}$, and a thiocarbonyl band at $1149-1194 \mathrm{~cm}^{-1}$ in addition to a carbonyl absorption at $1650-1661 \mathrm{~cm}^{-1}$. The structures of the above compounds were further confirmed by their ${ }^{1} \mathrm{H}-\mathrm{NMR},{ }^{13} \mathrm{C}$ NMR, and MS data. The fluorination of $\mathbf{3 a}$ by boiling with trifluoroacetic anhydride afforded 1,3-disubstituted5-trifluoroacetyl-thiobarbituric acid 4 (Scheme 1). The IR spectrum showed beside the $\mathrm{HN}$ and $\mathrm{OH}$ bands at 3103 and 3310, two carbonyl absorption bands at 1671 and 1714 in addition to thiocarbonyl band at $1181 \mathrm{~cm}^{-1}$. The mass fragmentation pattern of compound $\mathbf{4}$ is shown in Figure 2. The structure of 4 was further confirmed from its ${ }^{1} \mathrm{H}-\mathrm{NMR}$ and ${ }^{13} \mathrm{C}$ NMR data.

Careful treatment of PAS with $\mathrm{CS}_{2}$ (DMF) and trifluoroacetic anhydride in THF afforded 4-dithiocarboxyamino2-hydroxybenzoic acid 9a and 2-hydroxy-4-trifluoroacetyl amino benzoic acid $9 \mathbf{b}$, respectively. Moreover, ring closure reaction of $9 \mathbf{a}$ by refluxing it with chloroacetic acid in DMF produced 2-hydroxy-4-(4-oxo-2-thioxothiazolidin-3yl)benzoic acid 10 (Scheme 2). The IR spectrum of 9 a showed two absorption bands at $1800 \mathrm{~cm}^{-1}$ and $1284 \mathrm{~cm}^{-1}$ due to the $\mathrm{CO}$ and CS groups, respectively, while the IR spectrum of $\mathbf{9 b}$ exhibited two carbonyl bands at $1701 \mathrm{~cm}^{-1}$ and $1660 \mathrm{~cm}^{-1}$. The structures of $\mathbf{9 a}$ and $\mathbf{9 b}$ were further confirmed from their MS analyses which exhibited molecular ion peaks at $\mathrm{m} / \mathrm{z} 230$ and $\mathrm{m} / \mathrm{z} 250$, respectively (Figure 3 ).

Refluxing 8c with chloroacetic acid and sodium acetate in ethanol under neutral conditions furnished 2-hydroxy-4-(4 -oxo-3-p-methoxyphenylthiazolidin-2-ylideneamino) benzoic acid 11 (Scheme 2). The IR spectrum showed cyclic carbonyl absorption at $1724 \mathrm{~cm}^{-1}$ as well as $\mathrm{OH}$ absorption band at $3300 \mathrm{~cm}^{-1}$. The structure was further supported by its ${ }^{13} \mathrm{C}$ NMR data. 
Wadher et al. reported the synthesis of 4-thiazolidinone derivatives of $\mathrm{p}$-Aminosalicylic acid which showed in vitro a significant antimicrobial activity [19]. We have further extended this work by refluxing PAS with potassium dithiocarbazinate 12 in $\mathrm{EtOH}$ to yield $\mathrm{N}^{1}-\left(4^{\prime}\right.$-pyridinoyl)- $\mathrm{N}^{4}$ (2-hydroxybenzoic acid)thiosemicarbazide 13. The compounds 9 and $\mathbf{1 3}$ has analogous mass fragmentation patterns (Figure 4).

The cyclization of $\mathbf{1 3}$ in alkaline medium by refluxing it with aqueous $\mathrm{NaOH}$ produced 2-hydroxy-4-(3-(pyridin4-yl)-5-thioxo-1H-1,2,4-triazol-4(5H)-yl) benzoic acid 14. However, the cyclization in the acidic medium using cold $\mathrm{H}_{2} \mathrm{SO}_{4}$ at room temperature, resulted in the formation of 2-hydroxy-4-(5-pyridin-4-yl-[1,3,4] thiadiazol2-ylamino)benzoic acid 15 (Scheme 3 ). The structures of compounds 13-15 were characterized by IR, ${ }^{1} \mathrm{H}$, and ${ }^{13} \mathrm{C}$ NMR as well as by their mass fragmentation patterns.

3.2. Antimycobacterial Activity. All the new compounds obtained were tested for in vitro antituberculosis activity against $M$. tuberculosis $\mathrm{H} 37 \mathrm{Rv}$ using the BACTEC $12 \beta$ medium using a broth microdilution assay, the microplate alamar blue assay (MABA) [20, 21]. Rifampicin was used as the standard (Table 1). The antitubercular activities of the synthesized compounds were also investigated against M. fortutium ATCC6841, a rapidly growing strain, by the use of microdilution broth susceptibility methods [22-25]. Lowenstein-Jensen egg Medium and DMF for control purposes were used at $37^{\circ} \mathrm{C}$. Of these compounds, the ones which exhibited $>90 \%$ inhibition in the primary screen $(\mathrm{MIC}>$ $12 \mu \mathrm{g} / \mathrm{mL}$ ) were considered for further evaluation in the Level 2 of the screening (Table 2).

Compounds 2-4, 6, 8, 9, and 13 effecting 80-90\% inhibition in the primary screen at a concentration of $12.5 \mu \mathrm{g} / \mathrm{mL}$ were retested at lower concentrations against $M$. tuberculosis $\mathrm{H} 37 \mathrm{Rv}$ to determine the actual MIC (Table 2). The compound, $\mathbf{4}$ and $\mathbf{1 4}$ have shown a marked improvement in the antitubercular activity. In this case it has been demonstrated that substituted PAS may be of interest as antibacterial where the incorporation of isoniazid (INH) and $p$-Aminosalicylic acid in a simple molecule was aimed to enhance possible antitubercular activity which might arise from each side. Moreover, bulky groups introduced at nitrogen atom of PAS led to a decrease in activity which may be attributed to a steric hindrance and that causes an increased lipophilicity of this system [26-33].

From the preliminary data obtained from the level 1 screening, it was also found that compounds 2, 3, and 68 showed moderate activity against $M$. fortuitum. However, compounds 4, 9b, and 13-15 showed weak activity against the same strain.

\section{Conclusion}

p-Aminosalicylic acid is an important second-line treatment against mycobacterium as it may prevent the emergence of resistance to other antimycobacterial agents and enhances the efficacy of first-line drug isoniazid. Incorporation of structural features from both structures has seemingly improved the activity profiles in compound, 4 and 14. From the previously data, we can infer that both PAS bearing thiourea, thiobarbituric acids, and mercapto-1,2,4-triazole contributed significantly to exhibit the antitubercular activity. Compounds 4 and 14 were selected for further structure optimization.

\section{Acknowledgments}

The project was funded by the Deanship of Scientific Research (DSR), King Abdulaziz University, Jeddah, under Grant no. 561/130/1431. The authors, therefore, acknowledge with thanks DSR technical and financial support.

\section{References}

[1] A. H. Bacelar, M. A. Carvalho, and M. F. Proença, "Synthesis and in vitro evaluation of substituted pyrimido[5,4d]pyrimidines as a novel class of Antimycobacterium tuberculosis agents," European Journal of Medicinal Chemistry, vol. 45, no. 7, pp. 3234-3239, 2010.

[2] C. Dye, "Doomsday postponed? Preventing and reversing epidemics of drug-resistant tuberculosis," Nature Reviews Microbiology, vol. 7, no. 1, pp. 81-87, 2009.

[3] A. M. Ginsberg, "Emerging-drugs for active Tuberculosis," Seminars in Respiratory and Critical Care Medicine, vol. 29, no. 5, pp. 552-559, 2008.

[4] H. D. H. Showalter and W. A. Denny, "A roadmap for drug discovery and its translation to small molecule agents in clinical development for tuberculosis treatment," Tuberculosis, vol. 88, supplement 1, pp. S3-S17, 2008.

[5] H. Tomioka, Y. Tatano, K. Yasumoto, and T. Shimizu, "Recent advances in anti-tuberculous drug development and novel drug targets," Expert Review of Respiratory Medicine, vol. 2, no. 4, pp. 455-471, 2008.

[6] R. C. Goldman, K. V. Plumley, and B. E. Laughon, "The evolution of extensively drug resistant tuberculosis (XDRTB): history, status and issues for global control," Infectious Disorders, vol. 7, no. 2, pp. 73-91, 2007.

[7] L. Nguyen and C. J. Thompson, "Foundations of antibiotic resistance in bacterial physiology: the mycobacterial paradigm," Trends in Microbiology, vol. 14, no. 7, pp. 304-312, 2006.

[8] I. Küçükgüzel, E. Tatar, S. G. Küçükgüzel, S. Rollas, and E. De Clercq, "Synthesis of some novel thiourea derivatives obtained from 5-[(4-aminophenoxy)methyl]-4-alkyl/aryl2,4-dihydro-3H-1,2,4-triazole-3-thiones and evaluation as antiviral/anti-HIV and anti-tuberculosis agents," European Journal of Medicinal Chemistry, vol. 43, no. 2, pp. 381-392, 2008.

[9] J. Rawat, P. K. Jain, V. Ravichandran, and R. K. Agrawal, "Synthesis and evaluation of mutual prodrugs of isoniazid, p-amino salicylic acid and ethambutol," Archive for Organic Chemistry, vol. 2007, no. 1, pp. 105-118, 2007.

[10] M. Shiradkar, G. V. Suresh Kumar, V. Dasari, S. Tatikonda, K. C. Akula, and R. Shah, "Clubbed triazoles: a novel approach to antitubercular drugs," European Journal of Medicinal Chemistry, vol. 42, no. 6, pp. 807-816, 2007.

[11] B. Zhou, Y. He, X. Zhang et al., "Targeting mycobacterium protein tyrosine phosphatase B for antituberculosis agents," 
Proceedings of the National Academy of Sciences of the United States of America, vol. 107, pp. 4573-4578, 2010.

[12] R. M. Abdel-Rahman, K. O. Al-Footy, and F. M. Aqlan, "Synthesis and antiinflammatory evaluation of some more new 1,2,4triazolo[3,4-b] thiadiazoles as an antimicrobial agent-part 1," International Journal of ChemTech Research, vol. 3, no. 1, pp. 423-434, 2011.

[13] R. M. Abdel-Rahman, M. S. I. T. Makki, and W. A. Bawazir, "Synthesis of some more fluorine heterocyclic nitrogen systems derived from sulfa drugs as photochemical probe agents for inhibition of vitiligo disease-part i," E-Journal of Chemistry, vol. 8, no. 1, pp. 405-414, 2011.

[14] R. M. Abdel-Rahman, M. S. I. T. Makki, and W. A. B. Bawazir, "Synthesis of fluorine heterocyclic nitrogen systems derived from sulfa drugs as photochemical probe agents for inhibition of vitiligo disease-Part II," E-Journal of Chemistry, vol. 7, no. 1, pp. S93-S102, 2010.

[15] T. E. Ali, R. M. Abdel-Rahman, F. I. Hanafy, and S. M. ElEdfawy, "Synthesis and molluscicidal activity of phosphoruscontaining heterocyclic compounds derived from 5,6-bis (4bromophenyl)-3-hydrazino-1,2,4-triazine," Phosphorus, Sulfur and Silicon and the Related Elements, vol. 183, no. 10, pp. 2565-2577, 2008.

[16] R. M. Abdel-Rahman, "Chemistry of uncondensed 1,2,4triazines, part iv synthesis and chemistry of bioactive 3amino-1,2,4-triazines and related compounds - An overview," Pharmazie, vol. 56, no. 4, pp. 275-286, 2001.

[17] R. M. Abdel-Rahman, "Role of uncondensed 1,2,4-triazine compounds and related heterobicyclic systems as therapeutic agents-a review," Pharmazie, vol. 56, no. 1, pp. 18-22, 2001.

[18] R. M. Abdel-Rahman, "Role of uncondensed 1,2,4-triazine derivatives as biocidal plant protection agents-a review," Pharmazie, vol. 56, no. 3, pp. 195-204, 2001.

[19] S. J. Wadher, N. A. Karande, S. D. Sonawane, and P. G. Yeole, "Synthesis and biological evaluation of schiff base and 4-thiazolidinones of amino salicylic acid and their derivatives as an antimicrobial agent," International Journal of ChemTech Research, vol. 1, no. 4, pp. 1303-1307, 2009.

[20] W. Zheng, Y. M. Jiang, Y. Zhang, W. Jiang, X. Wang, and D. M. Cowan, "Chelation therapy of manganese intoxication with para-aminosalicylic acid (PAS) in Sprague-Dawley rats," NeuroToxicology, vol. 30, no. 2, pp. 240-248, 2009.

[21] V. Mathys, R. Wintjens, P. Lefevre et al., "Molecular genetics of para-aminosalicylic acid resistance in clinical isolates and spontaneous mutants of Mycobacterium tuberculosis," Antimicrobial Agents and Chemotherapy, vol. 53, no. 5, pp. 2100-2109, 2009.

[22] J. E. Hawkins, R. J. Wallace, and B. A. Brown, "Antimicrobial susceptibility tests: mycobacteria," in Manual of Clinical Microbiology, A. Balows, W. J. Hausler, K. L. Herrmann, H. O. Isenberg, and H. J. Shadomy, Eds., pp. 1138-1152, American Society for Microbiology, Washington, DC, USA, 5th edition, 1991.

[23] NCCLS Methods for Dilution Antimicrobial Susceptibility Tests for Bacteria that Grow Aerobically, Approved Standard NCCLS document M7-A2 NCCLS, Villanova, Pa, USA, 2nd edition, 1990.

[24] J. M. Swenson, C. Thornsberry, and V. A. Silcox, "Rapidly growing mycobacteria: testing of susceptibility to 34 antimicrobial agents by broth microdilution," Antimicrobial Agents and Chemotherapy, vol. 22, no. 2, pp. 186-192, 1982.
[25] "NCCLS Performance Standards for Antimicrobial Susceptibility Testing," 3rd International Supplement M 100-S3, NCCLS, Villanova, Pa, USA, 1991.

[26] J. W. Wilson, P. Kelkar, and E. Frigas, "Para-aminosalicylic acid (PAS) desensitization review in a case of multidrug-resistant pulmonary tuberculosis," International Journal of Tuberculosis and Lung Disease, vol. 7, no. 5, pp. 493-497, 2003.

[27] N. Tsapis, D. Bennett, K. O’Driscoll et al., "Direct lung delivery of para-aminosalicylic acid by aerosol particles," Tuberculosis, vol. 83, no. 6, pp. 379-385, 2003.

[28] C. Tuleu, A. W. Basit, W. A. Waddington, P. J. Ell, and J. M. Newton, "Colonic delivery of 4-aminosalicylic acid using amylose-ethylcellulose-coated hydroxypropylmethylcellulose capsules," Alimentary Pharmacology and Therapeutics, vol. 16, no. 10, pp. 1771-1779, 2002.

[29] A. Kumar and S. K. Menon, "Fullerene derivatized s-triazine analogues as antimicrobial agents," European Journal of Medicinal Chemistry, vol. 44, no. 5, pp. 2178-2183, 2009.

[30] N. Ulusoy, A. Gürsoy, and G. Otuk, "Synthesis and antimicrobial activity of some 1,2,4-triazole-3-mercaptoacetic acid derivatives," Farmaco, vol. 56, no. 12, pp. 947-952, 2001.

[31] I. Küçükgüzel, S. Güniz Küçükgüzel, S. Rollas, and M. Kiraz, "Some 3-thioxo/alkylthio-1,2,4-triazoles with a substituted thiourea moiety as possible antimycobacterials," Bioorganic and Medicinal Chemistry Letters, vol. 11, no. 13, pp. 1703-1707, 2001.

[32] L. A. Collins and S. G. Franzblau, "Microplate Alamar blue assay versus BACTEC 460 system for high- throughput screening of compounds against Mycobacterium tuberculosis and Mycobacterium avium," Antimicrobial Agents and Chemotherapy, vol. 41, pp. 1004-1009, 1997.

[33] S. G. Küçükgüzel, S. Rollas, I. Küçükgüzel, and M. Kiraz, "Synthesis and antimycobacterial activity of some coupling products from 4-aminobenzoic acid hydrazones," European Journal of Medicinal Chemistry, vol. 34, pp. 1093-1100, 1999. 

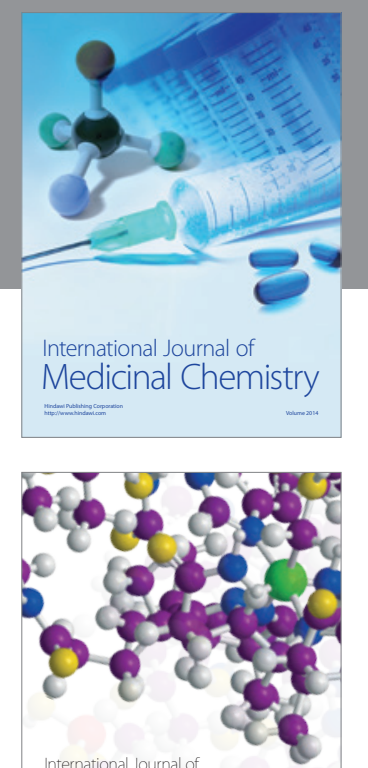

\section{Carbohydrate} Chemistry

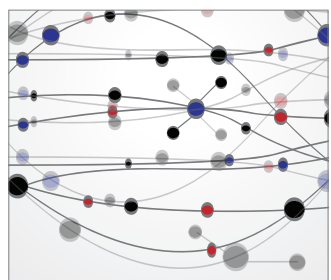

The Scientific World Journal
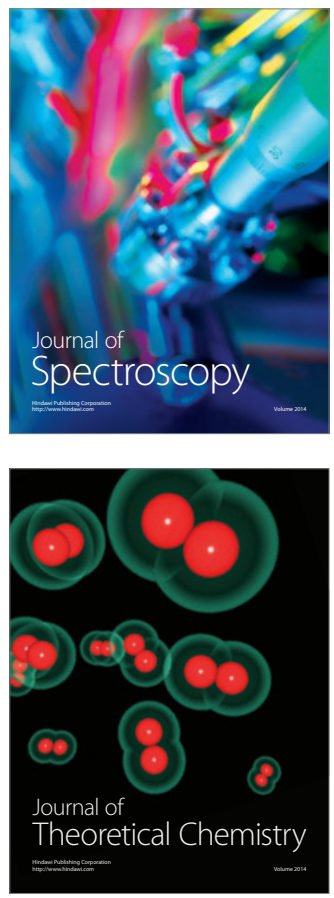
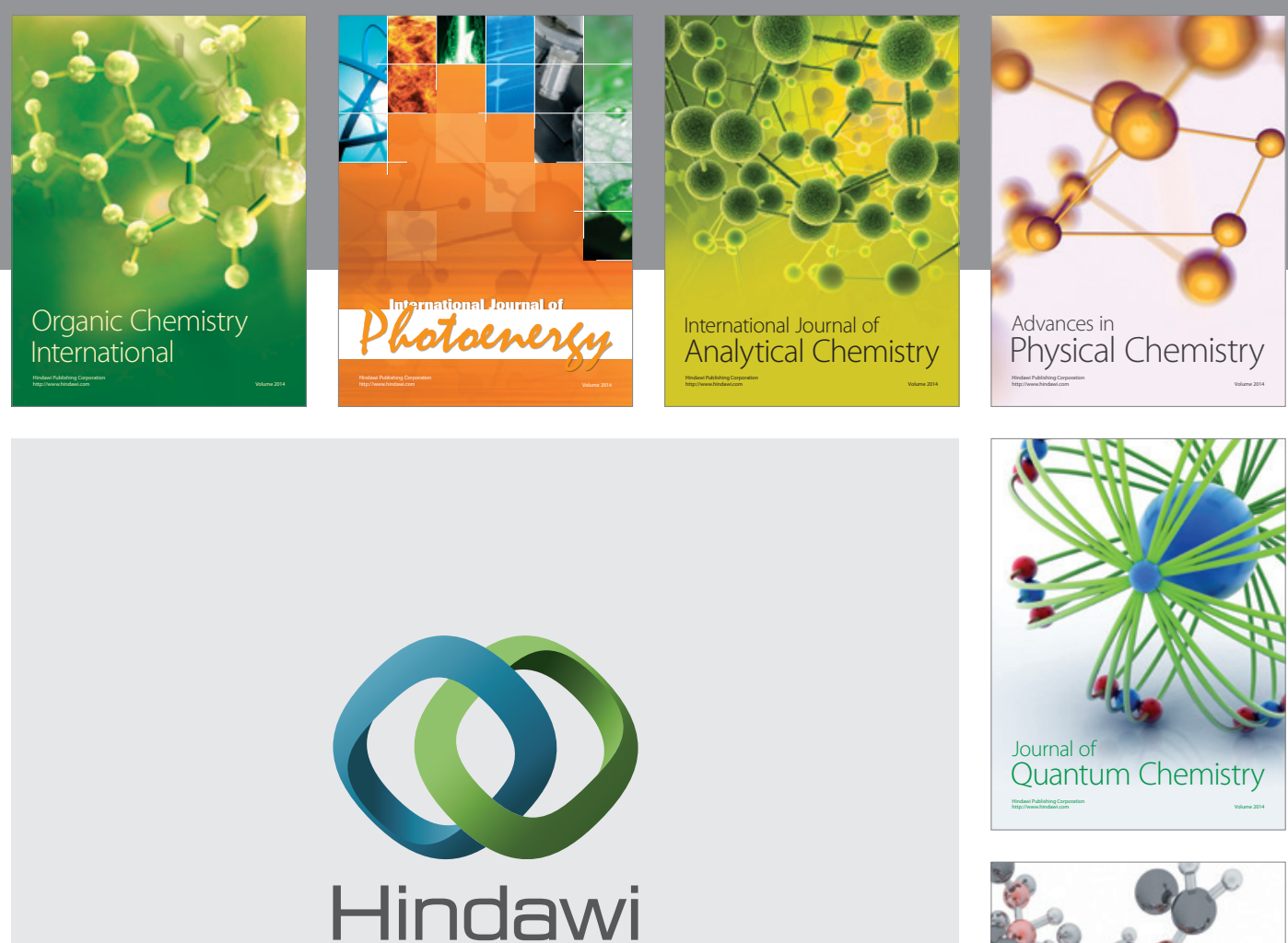

Submit your manuscripts at

http://www.hindawi.com

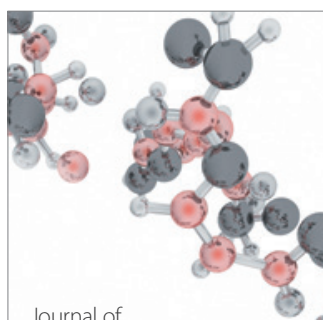

Analytical Methods

in Chemistry

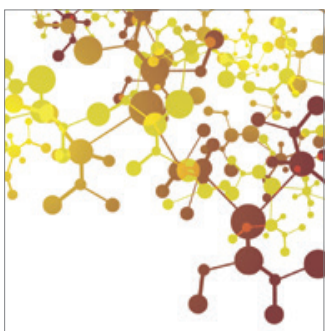

Journal of

Applied Chemistry

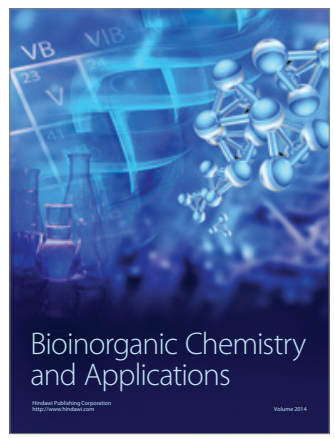

Inorganic Chemistry
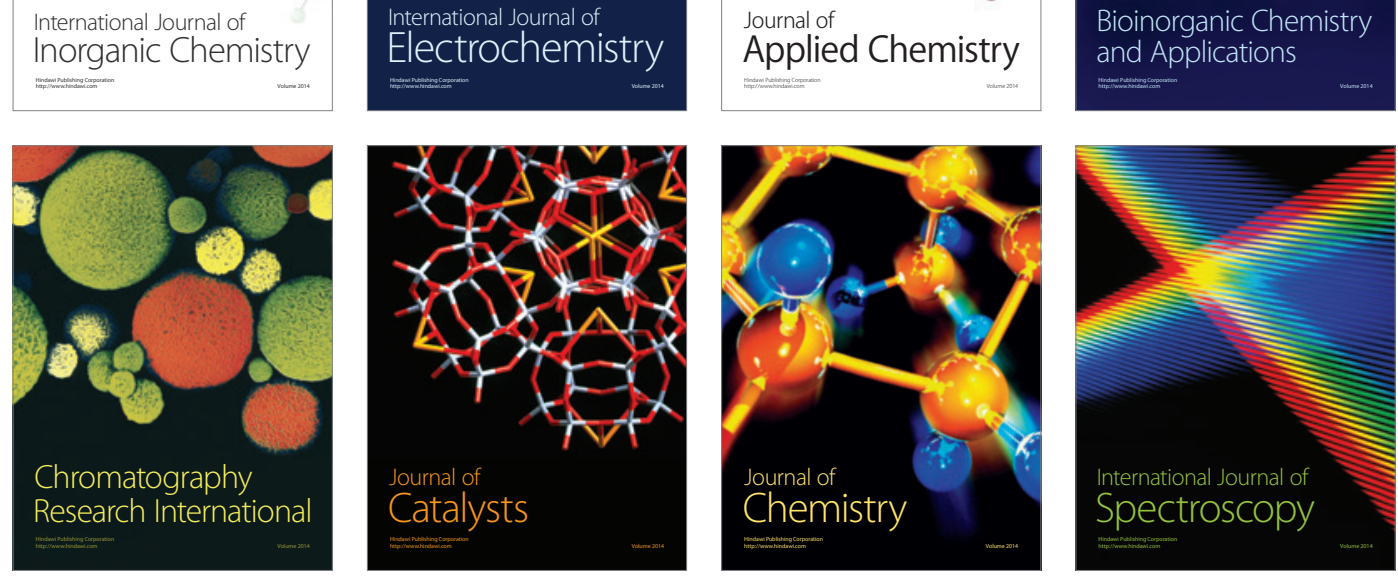